\title{
Die ACUITY-Studie
}

\section{Acute Catheterization and Urgent Intervention Triage strategY}

Hintergrund und Problemstellung: Bei Patienten mit akutem Koronarsyndrom ohne ST-Strecken-Hebung (NSTE-ACS) und hohem thrombotischen Risiko gilt heute die frühe invasive Strategie als gesichert. Im Vordergrund der medikamentösen Begleittherapie stehen - neben ASS und Clopidogrel - indirekte Antithrombine (unfraktioniertes Heparin = UFH; niedermolekulare Heparine = LMWH) und Glykoprotein-IIb/IIIa-Rezeptor-Inhibitoren (GPI). Das direkte Antithrombin Bivalirudin stellt eine mögliche Alternative zu den

\begin{tabular}{|c|c|}
\hline Methodik & \\
\hline Studiendesign & Randomisiert, kontrolliert, offen \\
\hline Primärer Endpunkt & $\begin{array}{l}\text { Kombinierter klinischer Endpunkt aus isch- } \\
\text { ämischen Ereignissen (Tod, Myokardinfarkt, un- } \\
\text { geplante PCI) und größeren Blutungen (nicht } \\
\text { im Rahmen einer Bypass-OP) nach } 30 \text { Tagen }\end{array}$ \\
\hline Patientenauswahl & $\begin{array}{l}\text { Einschlusskriterien: Brustschmerzen } \geq 10 \mathrm{~min} \\
<24 \mathrm{~h}, \\
\text { mindestens einer der folgenden Parameter: } \\
\text { - neu aufgetretene ST-Strecken-Senkung oder } \\
\text { transiente ST-Strecken-Hebung } \geq 1 \mathrm{~mm} \\
\text { - Troponin positiv } \\
\text { - dokumentierte KHK } \\
\text { - andere } 4 \text { TIMI-Risikokriterien } \\
\text { Ausschlusskriterien: kein Herzkatheter }<72 \mathrm{~h} \text {; } \\
\text { STEMI; kardiogener Schock; größere } \\
\text { Blutungen }\end{array}$ \\
\hline $\begin{array}{l}\text { Anzahl der teil- } \\
\text { nehmenden Zentren }\end{array}$ & 448 Zentren in 17 Staaten \\
\hline
\end{tabular}

Ergebnisse (* $p<0,05$ für Überlegenheit)

\begin{tabular}{|c|c|c|c|}
\hline & $\begin{array}{l}\text { Heparine } \\
\text { + GPI: }\end{array}$ & $\begin{array}{l}\text { Bivalirudin } \\
\text { + GPI: }\end{array}$ & $\begin{array}{l}\text { Bivalirudin } \\
\text { allein }\end{array}$ \\
\hline Patientenzahl & $n=4603$ & $n=4604$ & $\mathrm{n}=4612$ \\
\hline $\begin{array}{l}\text { Ausgangsdaten: } \\
\text { Patienten- } \\
\text { charakteristika }\end{array}$ & \multicolumn{3}{|c|}{$\begin{array}{l}\text { Alle } 3 \text { Gruppen waren in den demographischen } \\
\text { Basisdaten vergleichbar, ebenso in den Biomar- } \\
\text { kern und TIMI-Risikoscores. Die Angiographie- } \\
\text { raten lagen bei } 99 \% .56 \% \text { der Patienten wurden } \\
\text { dilatiert, } 11 \% \text { Bypass-operiert }\end{array}$} \\
\hline $\begin{array}{l}\text { Primärer Endpunkt } \\
\text { (s. o.) }\end{array}$ & $11,7 \%$ & $11,8 \%$ & $10,1 \%$ * \\
\hline Ischämische Ereignisse & $7,3 \%$ & $7,7 \%$ & $7,8 \%$ \\
\hline Größere Blutungen & $5,7 \%$ & $5,3 \%$ & $3,0 \%$ * \\
\hline - intrakraniell & $0,07 \%$ & $0,04 \%$ & $0,07 \%$ \\
\hline - retroperitoneal & $0,5 \%$ & $0,6 \%$ & $0,2 \%$ * \\
\hline $\begin{array}{c}\text { - im Bereich des } \\
\text { Gefäßzugangs }\end{array}$ & $2,6 \%$ & $2,6 \%$ & $0,8 \% *$ \\
\hline Hämatom $\geq 5 \mathrm{~cm}$ & $2,2 \%$ & $2,2 \%$ & $0,7 \% *$ \\
\hline Tod & $1,3 \%$ & $1,5 \%$ & $1,6 \%$ \\
\hline Myokardinfarkt & $4,9 \%$ & $5,0 \%$ & $5,4 \%$ \\
\hline Ungeplante PCI & $2,3 \%$ & $2,7 \%$ & $2,4 \%$ \\
\hline
\end{tabular}

genannten Substanzen dar, wurde aber noch nicht prospektiv in einer dem heutigen PCI-Standard entsprechenden Studie geprüft. Ziel der Studie war es festzustellen, ob im Rahmen einer geplanten PCI bei NSTE-ACS-Patienten mit erhöhtem thrombotischen Risiko eine Bivalirudin-Monotherapie im Vergleich zur Kombination Heparin (UFH/LMWH) + GPI oder zur Kombination Bivalirudin + GPI zu einem besseren klinischen Verlauf führt. Studienleiter war Gregg Stone, New York, USA.

\begin{tabular}{|c|c|c|}
\hline \multicolumn{3}{|c|}{ Evidence-based-Medicine- (EBM-) Score (www.TCTMD.com) } \\
\hline Klinischer primärer Endpunkt & $\mathrm{Ja}=3$, nein $=0$ & 3 \\
\hline Doppelblind (einschließlich Ärzten) & $\mathrm{Ja}=1$, nein $=0$ & 0 \\
\hline $\begin{array}{l}\text { Beobachtungsintervall für den } \\
\text { primären Endpunkt } \geq 6 \text { Monate }\end{array}$ & $\mathrm{Ja}=1$, nein $=0$ & 0 \\
\hline Multicenter (mindestens 3 Zentren) & $\mathrm{Ja}=1$, nein $=0$ & 1 \\
\hline $\begin{array}{l}\text { Externes und vom Steering } \\
\text { Committee unabhängiges Clinical } \\
\text { Event Committee / DSMB (Daten- } \\
\text { sicherheit Monitoring Board) }\end{array}$ & $\mathrm{Ja}=1$, nein $=0$ & 1 \\
\hline Primärer Endpunkt erreicht & $\mathrm{Ja}=1$, nein $=0$ & 1 \\
\hline $\begin{array}{l}\text { Power von } \geq 80 \% \text { für den } \\
\text { primären Endpunkt erreicht }\end{array}$ & $\mathrm{Ja}=1$, nein $=0$ & 1 \\
\hline $\begin{array}{l}\text { Anteil des Follow-up der Patienten } \\
\text { für angiographischen primären } \\
\text { Endpunkt } \geq 80 \% \text { und } \geq 95 \% \text { für } \\
\text { klinischen primären Endpunkt }\end{array}$ & $\mathrm{Ja}=1$, nein $=0$ & 1 \\
\hline \multicolumn{2}{|c|}{$\begin{array}{l}\text { Gesamt-EBM-Score } \\
\text { („Silber-Score“ für randomisierte Studien) }\end{array}$} & 8 \\
\hline
\end{tabular}

Schlussfolgerung und Kommentar: Bei Patienten mit NSTE-ACS und erhöhtem thrombotischen Risiko ist die Monotherapie mit Bivalirudin eine akzeptable Alternative zur Kombinationstherapie eines Heparins mit einem GPI. Der primäre Endpunkt einer signifikanten Besserung der klinischen Ereignisse nach 30 Tagen wurde erreicht. Dieser positive Effekt der Bivalirudin-Monotherapie beruhte vor allem auf einer signifikanten Reduktion von Blutungen im Bereich des arteriellen Zugangs. Der Effekt war unabhängig vom Alter und besonders bei Männern, Diabetikern, Patienten mit normalem Troponin, normaler ST-Strecke und bei Patienten mit Thienopyridin- (Clopidogrel-)Vorbehandlung signifikant. Unklar bleibt, weshalb die Überlegenheit der Bivalirudin-Monotherapie nur ausserhalb der USA, nicht aber in den USA nachgewiesen werden konnte. Nichtsdestoweniger liefert ACUITY die wissenschaftliche Grundlage, bei Patienten mit NSTE-ACS und geplanter Herzkatheteruntersuchung die Kombination eines Heparins mit einem Glykoprotein-IIb/IIIa-Rezeptorinhibitor durch die Bivalirudin-Monotherapie zu ersetzen.

Herz 2006;31:172

DOI $10.1007 /$ s00059-006-2814-x 